\title{
Гидротехническое строительство
}

DOI: http://www.dx.doi.org/10.24866/2227-6858/2020-2-15

УДК 626.01/004.942

\section{О.А. Макаров, А.Т. Беккер, Д.з. Гоголадзе}

МАКАРОВ ОЛЕГ АРТУРОВИЧ - магИстрант, мЛадший научныЙ сотрудник МНОЦ «R\&D центр «Арктика», ORCID: https://orcid.org/0000-0003-3319-4481, e-mail: makarov.oa@dvfu.ru БЕККЕР АЛЕКСАНДР ТЕВЬЕВИЧ - член-корреспондент РААСН, д.Т.Н., профрессор, научный руководитель Инженерной школы, SPIN: 3956-2515, ResercherID: I-6494-2017, ORCID: http://orcid.org/0000-0002-2899-7995, ScopusID: 35610298200, e-mail: bekker.at@dvfu.ru ГОГОЛАДЗЕ ДЕНИС ЗУРАБОВИЧ - магистрант, мЛадший научный сотрудник МНОЦ «R\&D центр «Арктика», ORCID: https://orcid.org/0000-0001-5497-807X, e-mail: gogoladze.dzu@dvfu.ru

Инженерная школа Дальневосточный фредеральный университет Владивосток, Россия

\section{Анализ конститутивных моделей пластичности применительно к численному моделированию ледовых воздействий}

Аннотация. В последние годы выполнено большое количество численных экспериментов для оценки величины воздействия льда на сооружения континентального шельфа с использованием различных моделей материалов. Однако корректных обоснований для применения таких моделей сравнительно мало. В связи с этим вопрос о достоверности характера поведения моделируемого льда, испытывающего значительные деформации, остается открытым. Хотя во многих программных комплексах имеется возможность интеграции сторонних моделей, созданных для описания поведения материалов под нагрузкой, использование этого инструмента требует от пользователя дополнительных знаний и навыков. В связи с этим в данной работе проведен анализ конститутивных моделей пластичности материала, имеющихся в базовых библиотеках программных комплексов ANSYS и SIMULIA Abaqus и учитывающих различные факторы, которые оказывают значительное влияние на прочностные характеристики льда. Для данных моделей выполнен анализ параметров, влияющих на критерий начала пластического течения. Сделан вывод о возможности и целесообразности применения рассмотренных моделей для различных условий. Проведен обзор численных исследований процессов взаимодействия в системе «лед-сооружение», выполненных различными авторами, в части применяемых конститутивных моделей.

Ключевые слова: конститутивные модели, ледовые воздействия, пластичность льда, скорость деформаций.

\section{Введение}

Применение численного моделирования сегодня - один из ведущих способов решения различных инженерных задач в области проектирования сооружений, в том числе гидротехнических сооружений (ГТС) на континентальном шельфе. С помощью различных программных 
комплексов решаются задачи структурной прочности, устойчивости, теплопроводности, моделируется поведение плавучих объектов в морской среде и т.п. Одна из важных задач при проектировании шельфовых ГТС ледовитых морей - оценка величины воздействия на них ледяных образований. Современные методы определения величины воздействия льда имеют большое количество допущений как со стороны применяемых параметров морского льда, так и с точки зрения комплексной картины взаимодействия «лёд-сооружение». Численное моделирование процесса взаимодействия сооружения с торосами, полями ровного льда и другими видами ледяных образований позволяет охватить значительное количество факторов и условий, влияющих на результирующую картину взаимодействия.

Одним из главных вопросов при моделировании процессов формирования нагрузок и воздействий ледяного покрова на ГТС является выбор конститутивных моделей льда как материала, описывающих его поведение на всех стадиях: упругая работа, пластическое поведение, развитие повреждений и полное разрушение. Правильный набор оптимальных моделей способствует наиболее точному формированию напряженно-деформированного состояния ледяных образований и сооружения, в том числе на контакте взаимодействующих тел.

Важность выбора оптимальной модели для льда как материала мы рассмотрим на примере следующих работ: Gürtner et al., 2009 [5], Gürtner et al., 2010 [4], Hilding et al., 2011 [6], Hilding et al., 2012 [7]. Эти исследования объединяет то, что авторы выполнили численные эксперименты воздействия ровного ледяного поля на конкретный объект - маяк Norströmsgrund, расположенный в Ботническом заливе. Маяк в 1999-2003 гг. в рамках проектов Low Level Ice Forces (LOLEIF) и Measurements on Structures in Ice (STRICE) оборудован специальными панелями, измеряющими ледовую нагрузку и покрывающими $167^{\circ}$ периметра сооружения. Кроме того, на маяке установлены специальные устройства для мониторинга условий окружающей среды. Таким образом выполнены полномасштабные натурные измерения ледовых нагрузок с контролем природных факторов, формирующих ледовый режим в акватории. В рассматриваемых работах авторы исследовали взаимодействие льда с сооружением при скорости дрейфа $0,15 \mathrm{~m} / \mathrm{c}$.

В качестве численного метода в этих работах использован метод конечных элементов с моделированием разрушения ледяного поля с помощью связных элементов. Основной принцип данного подхода заключается в моделировании ледяного поля в виде набора объемных конечных элементов, связанных между собой элементами практически нулевой толщины, являющимися по своей сути возможными путями образования и развития трещин (рис. 1 и рис. 2).

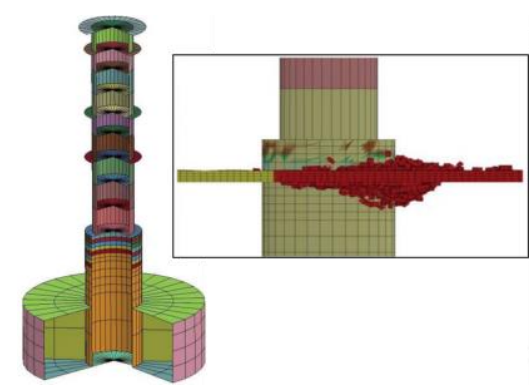

Рис. 1. Численная модель взаимодействия ледяного поля с сооружением в работе [4].

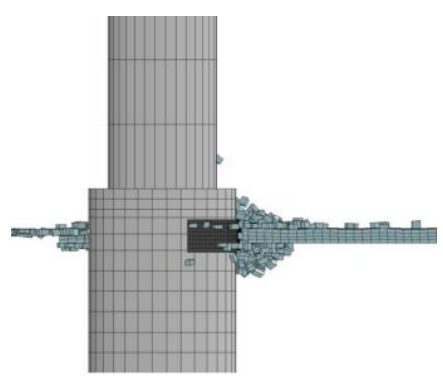

Рис. 2. Численная модель в работе [6].

Во всех перечисленных работах материал объемных элементов ледяного поля рассматривался как идеально-пластичный с использованием диаграммы Прандтля. При этом в качестве критерия пластичности использовалась модель Мизеса с поверхностью текучести, изображенной на рис. 3. Результаты моделирования, полученные в данных работах, достаточно адекватно описывают характер изменения ледовой нагрузки в процессе взаимодействия ледяного образо- 
вания и сооружением. Однако численные значения ледовой нагрузки существенно отличаются от измеренных в натурных условиях. В некоторых случаях расхождение в величине ледовой нагрузки на сооружение превышало 40\% (рис. 4)

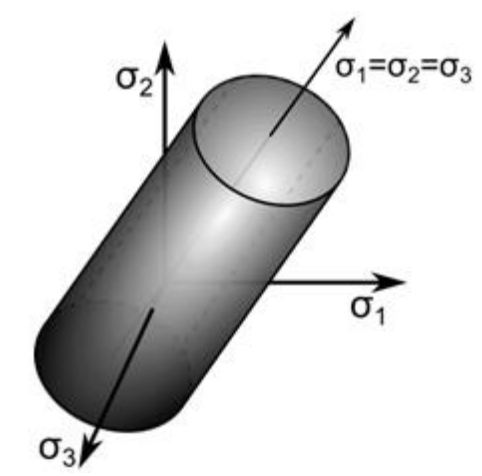

Рис. 3. Поверхность текучести для критерия Мизеса [3].

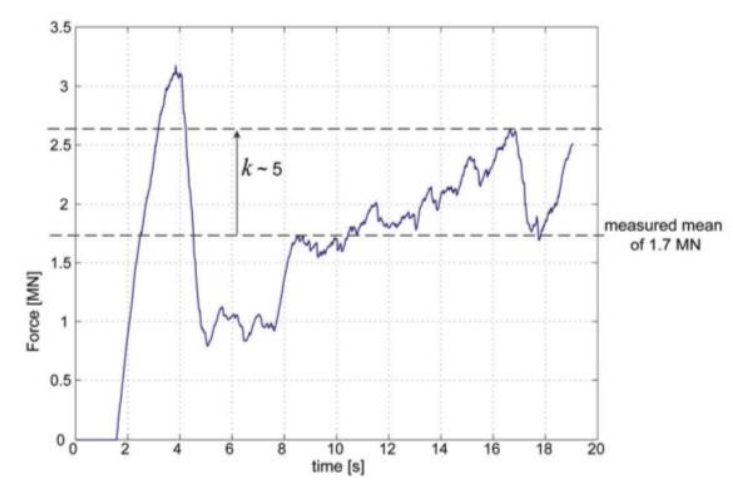

Рис. 4. Сравнение ледовой нагрузки, полученной в результате моделирования, с натурными данными в [5].

Хотя на результаты влияет множество факторов, по нашему мнению, авторами не был выполнен качественный подбор и дальнейшее обоснование принятой модели и параметров материала льда, что привело к большой степени несоответствия численного эксперимента и реальной картины взаимодействия.

Использование модели Мизеса приводит к введению большого количества допущений. Так, авторы не учитывали зависимость прочности льда от скорости деформаций, которая была описана ранее, а также пренебрегли температурными эффектами. Кроме того, как демонстрирует рис. 3, поверхность текучести Мизеса не имеет зависимости предела текучести от среднего гидростатического напряжения, что приводит к пренебрежению влияния сложного напряженно-деформированного состояния поля на общую прочность льда на контакте взаимодействия.

Значительная часть рассмотренных исследований по численному моделированию воздействия ледовых образований на шельфовые сооружения была проведена с помощью «тяжелых» программных комплексов ANSYS и SIMULIA Abaqus, имеющих широкую базу конститутивных моделей пластичности. Различные модели разрабатывались для описания поведения определенных материалов, например грунтов, металлов и бетона, широко применяемых в строительной области. Однако модели, полностью учитывающей особенности поведения под нагрузкой такого материала, как лед, на сегодняшний день не существует.

В программных комплексах имеется возможность интегрирования сторонних моделей через дополнительные инструменты, но это требует от пользователя более широких знаний, например в области программирования. В связи с этим цель настоящей статьи - выполнить анализ конститутивных моделей пластичности материала, имеющихся в базовых библиотеках программных комплексов ANSYS и SIMULIA Abaqus. Конститутивные модели мы рассмотрим с точки зрения учёта факторов, влияющих на параметры льда как материала: скорость деформаций, температура, среднее напряжение, а также неоднородность свойств в различных направлениях среды. Выбор данных пакетов программ обоснован их широким распространением и большим набором инструментов, предоставляющих возможности для решения соответствующих задач.

\section{Постановка задачи}

Сложность описания свойств льда связана в основном с их зависимостью от большого количества факторов. Так, на физико-механические характеристики льда оказывают существенное влияние структура поликристаллов льда, анизотропия, пространственная неоднород- 
ность ледяного покрова, сложное напряженное состояние, температура среды, наличие солевых включений, скорость деформаций и другие факторы. Если влияние температуры, солевых включений и, в какой-то степени, структуры могут быть учтены путем изменения коэффициентов матрицы жесткости материала, то учет влияния скорости деформаций и среднего напряжения на процесс деформации требует применения подходящих конститутивных моделей пластичности.

При рассмотрении влияния скорости деформаций на прочность льда и характер разрушения выделяют три основных зоны: зона пластичного поведения, переходная зона и зона хрупкого разрушения. Данная особенность неоднократно подтверждена натурными и лабораторными испытаниями, выполненными различными авторами (рис. 5).

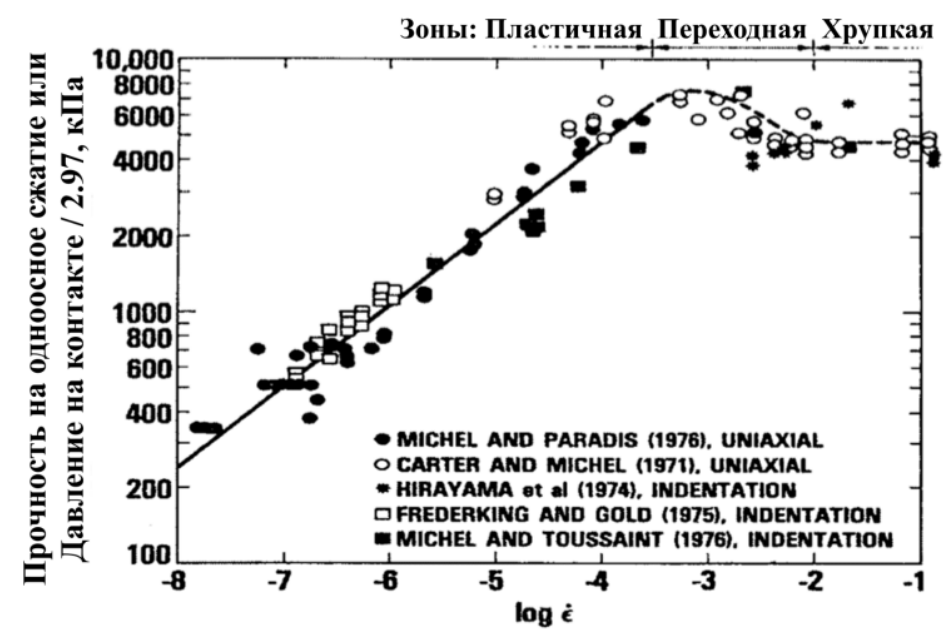

Рис 5. Характерная зависимость прочности льда от скорости деформаций [13].

Наиболее выраженные пластические свойства лёд проявляет в первой зоне, которая имеет значимость при малых скоростях взаимодействия. В данном случае можно наблюдать явные пластические деформации, связанные со взаимным смещением кристаллов льда [12]. Но поскольку наибольшее воздействие ледяных образований на шельфовые сооружения возникает при переходе от пластичного к хрупкому характеру разрушения, то именно переходная зона представляет наибольший интерес для практики. При относительно высоких скоростях деформаций $\left(\geq 10^{-3} \mathrm{c}^{-1}\right)$ лёд может рассматриваться как полностью линейно-упругий материал без развития пластических деформаций. В последнем случае модели пластичности могут служить моделями разрушения материала, при которых возникновение пластических деформаций выступает критерием разрушения. В связи с этим далее будут рассматриваться конститутивные модели, наиболее подходящие для описания процесса деформирования льда, соответствующего пластичной и переходной зонам.

Наиболее популярным методом исследования численного моделирования взаимодействия льда с сооружениями является метод конечных элементов в связи с его широким распространением в программных комплексах, а также относительной простотой использования и интерпретации результатов. Метод также использован в работах $[1,2,11]$, которые отличаются выбором конститутивных моделей, описанием физико-механических свойств льда, а также подходами к моделированию деградации и разрушения материала.

\section{Модели пластичности Друкера-Прагера}

Довольно широкое распространение в области моделирования сплошных сред имеют различные модели типа Друкера-Прагера. Данные модели деформирования материалов часто используются для моделирования гранулированных сред, например грунтов, а также хрупких материалов, таких как бетон. В инструментарии рассматриваемых программных комплексов 
имеется как классическая линейная модель, так и расширенные гиперболическая и экспоненциальная модели, имеющие, соответственно, следующие критерии текучести [15]:

$$
\begin{aligned}
& F=t-p \tan \beta-d=0, \\
& F=\sqrt{l_{0}^{2}+q^{2}}-p \tan \beta-d^{\prime}=0, \\
& F=a q^{b}-p-p_{t}=0,
\end{aligned}
$$

где $p$ - среднее (гидростатическое) напряжение; $p_{t}$ - предельное растягивающее среднее (гидростатическое) напряжение; $\beta$ - параметр материала, регулирующий наклон поверхности текучести к гидростатической оси и интерпретируемый обычно как угол внутреннего трения материала; $d$ - удельное сцепление материала; $q$ - эквивалентные напряжения по Мизесу; $a$ и $b$ - независимые параметры экспоненциальной модели; $t, l_{0}, d^{\prime}$ - автоматически определяемые параметры модели.

Формы кривых, образующих поверхности текучести для данных моделей, представлены на рис. 6. Как следует из (1)-(3), модели Друкера-Прагера учитывают влияние среднего напряжения на общую прочность материала, что является одним из основных факторов при оценке величины воздействия льда на сооружения. Стоит отметить, что с точки зрения процесса развития пластических деформаций в программном комплексе ANSYS не предусмотрен учёт упрочнения материала и, соответственно, изменение поверхности текучести в процессе расчета.

Напротив, в SIMULIA Abaqus присутствуют расширенные настройки данных моделей, в том числе изотропное упрочнение материала, зависящее от прочности на сжатие, растяжение или сдвиг, а также изменение предела текучести в зависимости от скорости деформаций и температуры. Закон пластического течения в данном программном комплексе для модели Друкера-Прагера не ассоциативный, с возможностью учёта дилатансии. Также доступна функция изменения формы поверхности текучести в девиаторной плоскости для линейной модели и подбора параметров модели путем аппроксимации результатов испытаний образцов материала.

a)

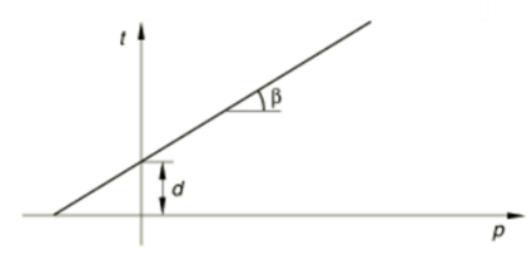

б)

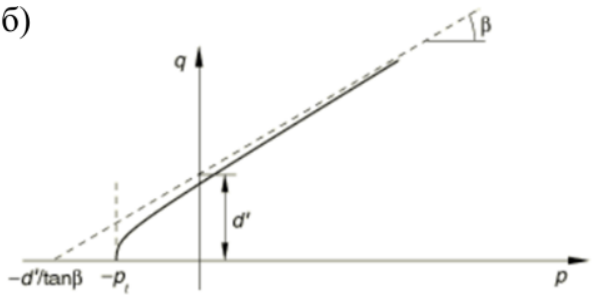

в)

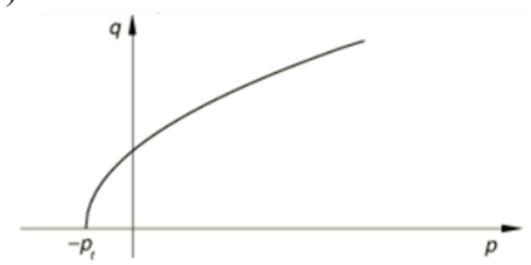

Рис. 6. Поверхности текучести моделей Друкера-Прагера:

a - линейная; б - гиперболическая; в - экспоненциальная [12]

Наиболее простой в применении с точки зрения подбора значений параметров является классическая линейная форма модели. В данном случае основными параметрами, определяемыми пользователем, являются угол внутреннего трения $\beta$, а также напряжения $\bar{\sigma}_{0}$, определяющие начальную форму поверхности текучести (при нулевых значениях пластических деформаций $\left.\bar{\varepsilon}^{p l}\right)$.

B программном комплексе ANSYS данные напряжения соответствуют нулевому значению гидростатического напряжения и, соответственно, могут интерпретироваться как удельное сцепление материала.

B SIMULIA Abaqus напряжения $\bar{\sigma}_{0}$, определяющие начальную поверхность текучести, а также эквивалентные напряжения $\bar{\sigma}$ с соответствующими значениями пластических деформаций $\bar{\varepsilon}^{p l}$, определяющие упрочнение, могут по выбору пользователя интерпретироваться как предел текучести при одноосном сжатии, растяжении или удельное сцепление. На первый взгляд, очевидно, что наиболее удобной функцией является определение упрочнения че- 
рез напряжения одноосного сжатия, так как в большинстве случаев данные о нелинейном характере деформирования льда получены авторами через соответствующие испытания образцов. В то же время для хрупких материалов, в том числе льда, сцепление может интерпретироваться как предел текучести на сдвиг, но при определении упрочнения через напряжения сжатия значение сцепления $d$ будет вычисляться в модели в зависимости от угла внутреннего трения $\beta$, определенного пользователем, по следующей формуле [15]:

$$
d=\left(1-\frac{1}{3} \tan \beta\right) \bar{\sigma} .
$$

Кроме того, угол внутреннего трения $\beta$ для хрупких материалов является условной характеристикой, и для такого материала, как лёд, выбор оптимального значения является трудной задачей. В связи с этим авторами данной статьи предлагается следующий алгоритм для моделирования льда с использованием линейной модели Друкера-Прагера в программном комплексе SIMULIA Abaqus.

1. Определение набора значений пределов прочности льда на одноосное сжатие $\sigma_{c}$ и сдвиг $\sigma_{\tau}$, соответствующих нескольким значениям температуры $t$ и скорости деформаций $\dot{\bar{\varepsilon}}^{p l}$. Данные могут быть получены по эмпирическим зависимостям, предложенным различными авторами, а также из результатов экспериментов.

2. Полагая, что $\bar{\sigma}=\sigma_{c}$, а $d=\sigma_{\tau}$, вычисление значений угла внутреннего трения $\beta$ по формуле:

$$
\beta=\tan ^{-1}\left(\frac{3 \cdot\left(\sigma_{c}-\sigma_{\tau}\right)}{\sigma_{c}}\right)
$$

Таким образом, форма поверхности текучести будет наиболее точно описывать прочностные характеристики моделируемого льда. Стоит отметить, что критерий ДрукераПрагера является изотропным (условный предел текучести одинаков во всех направлениях), поэтому его применение должно быть обосновано с точки зрения структуры моделируемого льда. Например, при описании свойств зернистого льда, который часто принимается условно изотропным, данное допущение, по мнению авторов, может быть оправдано. Однако при моделировании льда столбчатой структуры, который имеет существенные отличия прочности в различных направлениях, применение данной модели накладывает значительные ограничения и требует дополнительного обоснования

Из существующих исследований по моделированию взаимодействия ледяных образований и шельфовых сооружений, где для описания работы льда применялась модель пластичности Друкера-Прагера, можно выделить работу Sultabayev, 2015 [14]. Автор провел численные эксперименты при воздействии ровного ледяного поля на сооружение с наклонной гранью, в том числе с учетом скопившегося перед сооружением торошенного льда (рис. 7).

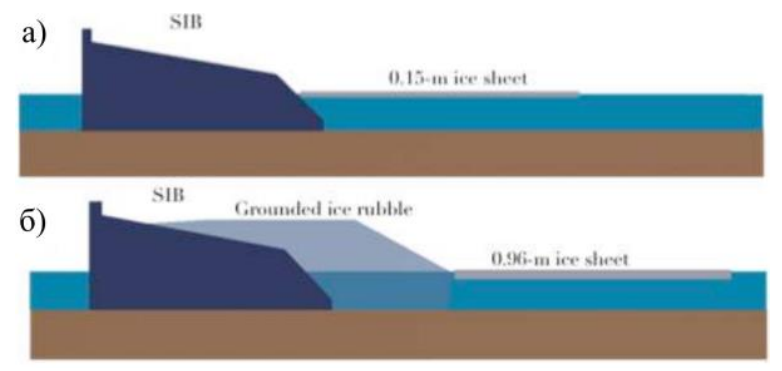

Рис. 7. Воздействие ровного ледяного поля на сооружение с наклонной гранью: а - при толщине льда 0,15 м; б - при толщине льда 0,96 м с учетом торошенного льда [14].

Результаты были проанализированы в части соответствия значения полученной при моделировании ледовой нагрузки значению, рассчитанному аналитическими методами. Разница величин составила более $40 \%$ в случае воздействия льда толщиной 0,15 м, и более $65 \%$ 
- толщиной 0,96 м. Однако аналитические методы также имеют свои допущения, поэтому, по нашему мнению, более целесообразно оценивать соответствие результатов моделирования данным полевых испытаний. Несмотря на то что автор [14] получил зависимости нагрузок от времени (рис. 8 и рис. 9), схожие по характеру с реальной картиной взаимодействия льда и сооружений, в работе не был произведен достаточно обоснованный подбор используемых параметров модели, а также её верификация. В связи с этим факт соответствия результатов моделирования натурным данным нельзя считать полностью корректным.

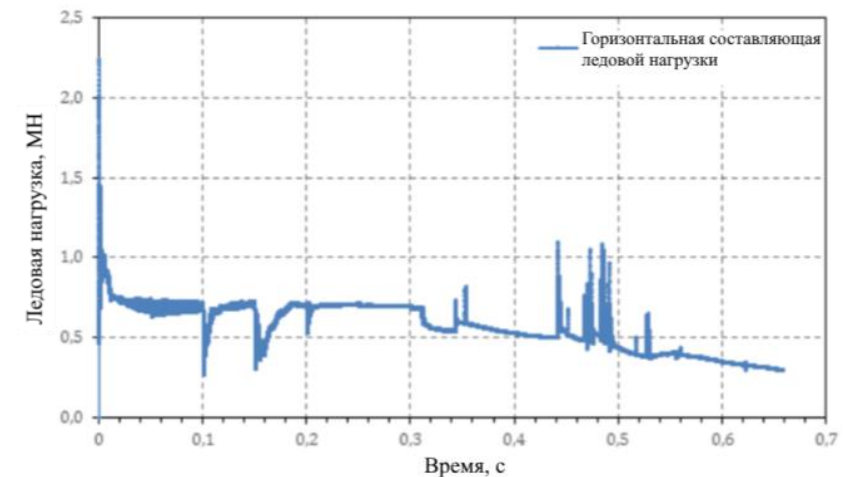

Рис. 8. График ледовой нагрузки в случае моделирования льда толщиной 0,15 м [14].

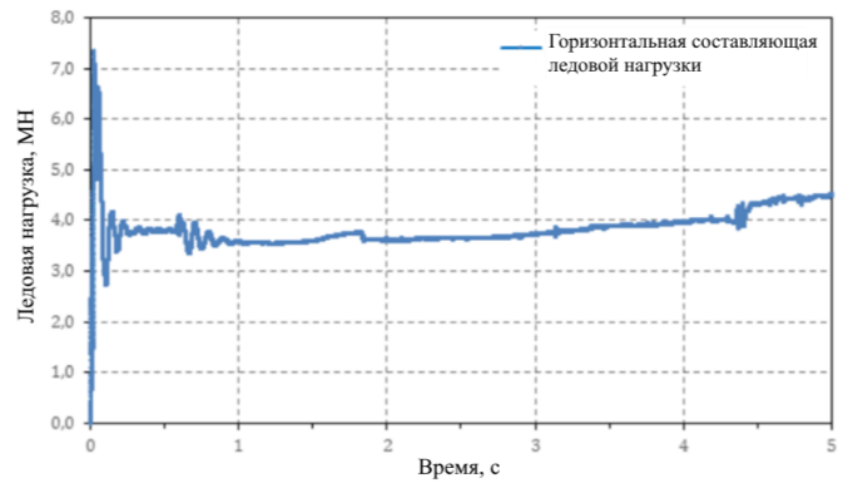

Рис. 9. График ледовой нагрузки в случае моделирования льда толщиной 0,96 м с учетом торошения [14].

\section{Модель пластичности Джонсона-Кука}

Особый интерес вызывает модель пластичности Джонсона-Кука, разработанная и описанная в 1985 г. Гордоном Джонсоном и Вильямом Куком [9], которая может подходить для моделирования поведения материалов, включая большинство металлов, при высоких деформациях, высоких скоростях деформаций, а также при температурах, близких к температурам плавления. Эти три фактора учитываются критерием текучести, который может быть описан следующим образом:

$$
\bar{\sigma}=\left[A+B\left(\bar{\varepsilon}^{p l}\right)^{n}\right] \cdot\left[1+C \ln \left(\frac{\dot{\bar{\varepsilon}}^{p l}}{\dot{\bar{\varepsilon}}_{0}^{p l}}\right)\right] \cdot\left(1-\hat{\theta}^{m}\right),
$$

где $\bar{\varepsilon}^{p l}$ - эквивалентные пластические деформации; $\dot{\bar{\varepsilon}}^{p l}-$ скорость эквивалентных пластических деформаций; $\dot{\bar{\varepsilon}}_{0}^{p l}$ - исходное значение скорости деформаций; $C$ - параметр материала, определяемый в условиях, не превышающих температуру перехода; $A$ - начальный предел текучести; $B$ и $n$ - коэффициенты, необходимые для описания упрочнения материала; $m-$ коэффициент термического размягчения; $\hat{\theta}$ - температурный параметр, зависящий от текущей температуры, температуры фазового перехода материала и определяемый по следующей формуле:

$$
\hat{\theta}=s_{i}=\left\{\begin{aligned}
0 & , \text { при } \theta<\theta_{\text {transition }} \\
\left(\theta-\theta_{\text {transition }}\right) /\left(\theta_{\text {melt }}-\theta_{\text {transition }}\right) & , \text { при } \theta_{\text {transition }} \leq \theta \leq \theta_{\text {melt }}, \\
1 & , \text { при } \theta>\theta_{\text {melt }}
\end{aligned}\right.
$$

где $\theta$ - текущая температура материала; $\theta_{\text {transition }}$ - температура перехода, ниже которой отсутствует зависимость предела текучести от температуры; $\theta_{\text {melt }}$ - температура плавления материала.

Из формулы (6) следует, что учет факторов, влияющих на начало пластического течения с ассоциативным законом, производится тремя множителями. В первом множителе описывается зависимость между величиной предела текучести и деформациями, т.е. изотропное упрочнение материала. Второй множитель учитывает влияние скорости деформаций на пре- 
дел текучести. Третье слагаемое характеризует эффект термического размягчения, при котором предел текучести становится равным нулю, если достигается температура плавления материала.

Стоит отметить, что поверхностью текучести в критерии Джонсона-Кука является поверхность Мизеса (рис. 3), не зависящая от среднего (гидростатического) напряжения и имеющая одинаковый предел текучести при сжатии и растяжении. Данные факты вносят значительные допущения при моделировании таких анизотропных материалов, как лед.

Применение этой модели, по нашему мнению, целесообразно только в случае моделирования ледовых воздействий в программном комплексе ANSYS, поскольку данная модель одна из немногих, имеющихся в его инструментарии, учитывает влияние скорости деформаций на величину предела текучести.

Однако, поскольку учет зависимости предела текучести от температуры в рассматриваемых программных комплексах возможен другими способами, а скорость деформации учитывается в SIMULIA Abaqus в других моделях (например, Друкера-Прагера), применение модели Джонсона-Кука для моделирования льда не рекомендуется.

Авторами не было найдено работ, в которых данная модель используется применительно ко льду. Однако в работе Nisja (2014) [10] проводилось исследование применимости различных конститутивных моделей, имеющихся в программном комплексе LS-DYNA, для численного моделирования воздействия льда на шельфовые сооружения. Одной из рассматриваемых моделей была модель Холмкиста-Джонсона-Кука, применяемая для моделирования бетона и других хрупких материалов. Критерий данной модели имеет следующий вид:

$$
\bar{\sigma}=\left[A \cdot(1-D)+B\left(\bar{\varepsilon}^{p l}\right)^{n}\right] \cdot\left[1+C \ln \left(\frac{\dot{\bar{\varepsilon}}^{p l}}{\dot{\bar{\varepsilon}}_{0}^{p l}}\right)\right] .
$$

Можно заметить значительную схожесть формулы (8) с формулой (6). Отличие составляет отсутствие третьего (температурного) множителя, а также наличие дополнительного параметра повреждения материала $D$, принимающего значения от 0 до 1 . В данной работе был проведен обоснованный подбор значений параметров, используемых в модели. Значения найденных коэффициентов можно принимать за исходные данные при подборе параметров для модели пластичности Джонсона-Кука.

Автором [10] проведены серии численных экспериментов воздействия ледяного поля на вертикальную опору (рис. 10) для сравнения рассматриваемых моделей материалов (рис. 11).

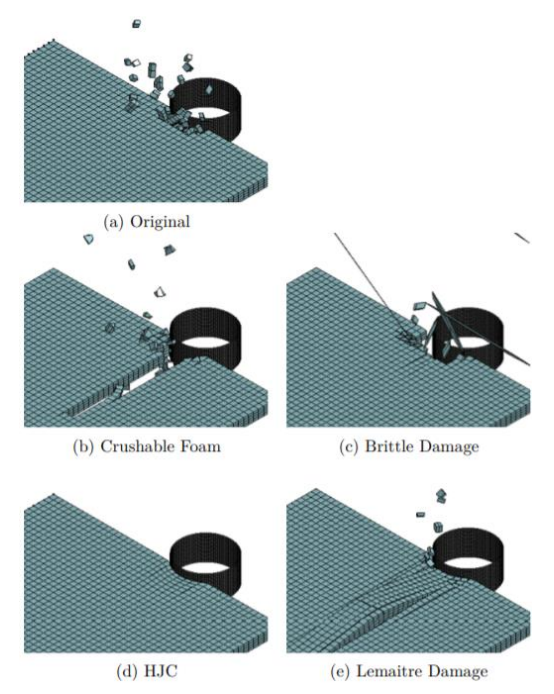

Рис. 10. Численное моделирование разрушения льда при воздействии на сооружение [10].

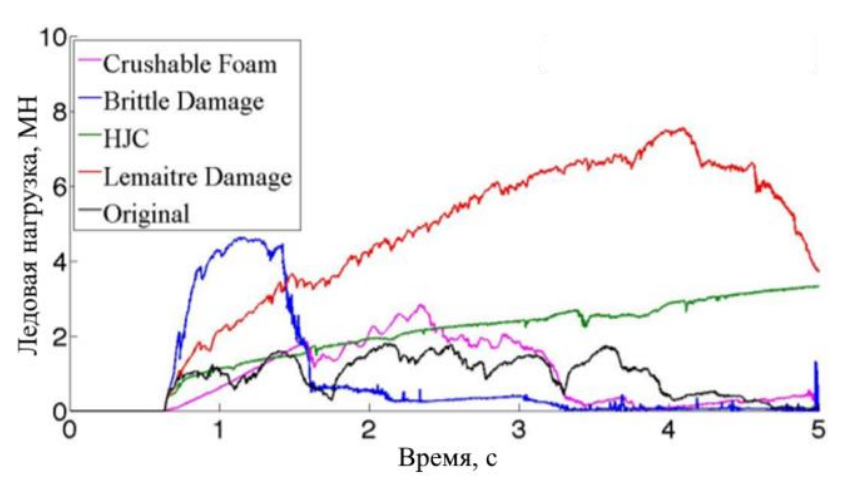

Рис. 11. Сравнение ледовой нагрузки при моделировании льда различными моделями [10]. 
Рисунок 11 показывает, что нагрузка, полученная с использованием модели Холмкиста-Джонсона-Кука, носит относительно плавный характер в отличие от результатов других моделей. Данный факт несколько противоречит реальной картине взаимодействия льда с сооружениями, что заставляет сомневаться в применимости модели Джонсона-Кука для моделирования льда.

\section{Модель пластичности Хилла}

Модель пластичности была разработана Родни Хиллом [8] для описания процесса анизотропного пластического деформирования таких материалов, как некоторые металлы, полимеры и композиты. В явном виде данная модель присутствует только в программном комплексе SIMULIA Abaqus, однако ортотропная модель пластичности в ANSYS Autodyn может быть приведена к модели Хилла, о чем будет сказано далее. Критерий текучести Хилла является простым расширением критерия Мизеса и имеет квадратичную форму:

$$
f(\sigma)=\sqrt{F\left(\sigma_{22}-\sigma_{33}\right)^{2}+G\left(\sigma_{33}-\sigma_{11}\right)^{2}+H\left(\sigma_{11}-\sigma_{22}\right)^{2}+2 L \sigma_{23}^{2}+2 M \sigma_{31}^{2}+2 N \sigma_{12}^{2}}=1,
$$

где $F, G, H, L, M, N$ - константы материала, определяемые в зависимости от коэффициентов текучести $R_{i j} ; \sigma_{11}, \sigma_{22}, \sigma_{33}, \sigma_{12}, \sigma_{23}, \sigma_{31}$ - пределы текучести в декартовой системе координат.

При этом значения пределов текучести $\sigma_{i j}$ не вводятся пользователем напрямую. Вместо этого в качестве исходных параметров для моделирования используются так называемые коэффициенты текучести $R_{i j}$, которые представляют собой отношение соответствующего значения предела текучести к исходному значению $\sigma_{0}$. Стоит отметить, что для вычисления коэффициентов касательных составляющих $R_{12}, R_{23}, R_{31}$ вместо $\sigma_{0}$ используется исходный предел текучести на сдвиг $\tau_{0}$ :

$$
\tau_{0}=\sigma_{0} / \sqrt{3},
$$

Как и критерий текучести Мизеса, основанный на нём квадратичный критерий Хилла не зависит от среднего (гидростатического) напряжения и предполагает одинаковое значение предела текучести на сжатие и растяжение. Данные особенности являются существенными недостатками модели при её применении для моделирования льда.

Среди преимуществ можно выделить простоту использования и возможность учёта различных значений предела текучести в трёх ортогональных направлениях. Кроме того, при применении модели Хилла в программном комплексе SIMULIA Abaqus есть возможность учёта упрочнения материала, а также зависимости предела прочности от температуры и скорости деформаций. Данные факты, наряду с малым количеством других ортотропных моделей в инструментарии программных комплексов, по нашему мнению, делают применение модели пластичности Хилла рациональным для моделирования льда с ярко выраженными ортотропными свойствами (например, столбчатого).

\section{Ортотропная модель пластичности}

Ортотропная модель пластичности в основном применяется для описания анизотропного упрочнения, наблюдаемого у некоторых композитных материалов. Данная модель доступна в программном комплексе ANSYS в модуле Autodyn. Поверхность текучести в данной модели определяется квадратичной функцией, которая может быть представлена в следующем виде:

$$
\begin{aligned}
& f\left(\sigma_{i j}\right)=a_{11} \sigma_{11}^{2}+a_{22} \sigma_{22}^{2}+a_{33} \sigma_{33}^{2}+2 a_{12} \sigma_{11} \sigma_{22}+2 a_{23} \sigma_{22} \sigma_{33}+2 a_{13} \sigma_{11} \sigma_{33}+ \\
& +2 a_{44} \sigma_{23}^{2}+2 a_{55} \sigma_{31}^{2}+2 a_{66} \sigma_{12}^{2}=k,
\end{aligned}
$$


где $a_{i j}$ - коэффициенты материала, регулирующие степень анизотропии материала; $\sigma_{11}, \sigma_{22}$, $\sigma_{33}, \sigma_{12}, \sigma_{23}, \sigma_{31}$ - пределы текучести в декартовой системе координат; $k$ - коэффициент, зависящий от неупругих деформаций и используемый для описания характера упрочнения.

Параметры модели определяются исходя из результатов испытаний материала на одноосное сжатие, одноосное растяжение и испытаний на чистый сдвиг. При этом шесть параметров $a_{11}, a_{22} a_{33}, a_{44}, a_{55}$ и $a_{66}$, связанных с нормальными напряжениями, выражаются из главных зависимостей эффективных напряжений-деформаций $\left(\bar{\sigma}-\bar{\varepsilon}^{p}\right)$, а также путем определения пластических коэффициентов Пуассона. Поскольку построение зависимостей «напряжения-деформации» для льда во всех направлениях является трудной задачей, имеет смысл использовать данный критерий как критерий разрушения материала. Тогда параметры модели могут быть легко определены из значений прочности льда на сжатие, растяжение и сдвиг, полученных экспериментально. Результаты таких экспериментов были опубликованы многими авторами для различных условий солености, пористости, температур и других факторов.

При соблюдении некоторых условий данная модель может быть приведена к модели пластичности Хилла, рассмотренной ранее, а также к классической модели Мизеса. После начала пластического течения поведение материала становится частично упругим и частично пластичным с ассоциативным законом течения. При этом материал может рассматриваться как идеально-пластичный, так и с кусочно-линейной функцией упрочнения.

Недостатком данной модели является отсутствие зависимости прочности материала от среднего напряжения, а также (как и во многих моделях комплекса ANSYS) - отсутствие зависимости от скорости деформаций. Однако возможность учёта разных прочностных свойств в ортогональных направлениях материала и отсутствие других ортотропных моделей материалов в ANSYS делают целесообразным использование данной модели при моделировании столбчатого льда.

Нами не было найдено каких-либо исследований нагрузок и воздействий льда на сооружения численными методами с использованием данной модели либо модели Хилла. Некоторые авторы (Sand, 2008 [11], Nisja [10] и др.) рассматривали возможность использования модели Хилла при моделировании столбчатого льда, однако отказались от её применения в связи с отсутствием зависимости предела текучести от среднего напряжения. Вместо этого, например Sand, использовал более подходящий в этом плане критерий Хоригмо и Зенга в качестве критерия разрушения, имеющего не только квадратичную зависимость от напряжений, но также и линейные компоненты:

$$
\begin{aligned}
& f\left(\sigma_{i j}\right)=a_{1} \sigma_{11}^{2}+a_{2} \sigma_{22}^{2}+a_{3} \sigma_{33}^{2}+a_{4} \sigma_{11} \sigma_{22}+a_{5} \sigma_{22} \sigma_{33}+a_{6} \sigma_{11} \sigma_{33}+a_{7} \sigma_{11}+ \\
& +a_{8} \sigma_{11}+a_{9} \sigma_{11}+a_{10} \sigma_{23}^{2}+a_{11} \sigma_{31}^{2}+a_{12} \sigma_{12}^{2}=1
\end{aligned}
$$

Очевидно, что данный критерий может быть легко приведен к критерию ортотропной модели, если принять $a_{8}=a_{9}=a_{10}=0$ и $k=1$. Поверхность текучести данного критерия изображена на рис. 12. С нашей точки зрения, данный критерий, несомненно, больше подходит для моделирования льда, чем модель Хилла и ортотропная модель пластичности. Однако данная модель не анализировалась более детально в связи с её отсутствием в инструментарии рассматриваемых программных комплексов. Автором [11] был выполнен и подбор значений коэффициентов $a_{i}$ путем аппроксимации результатов экспериментов, которые тоже могут быть использованы в ортотропной модели пластичности. 

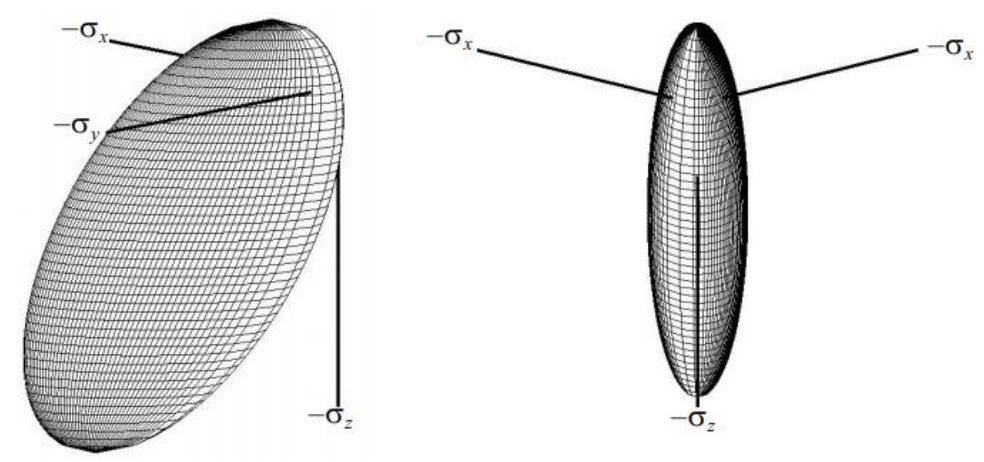

Рис. 12. Поверхность текучести критерия Хоригмо и Зенга [11].

\section{Заключение}

Анализ конститутивных моделей пластичности, доступных для использования в программных комплексах ANSYS и SIMULIA Abaqus, показал актуальность их применимости для моделирования поведения льда под нагрузкой. Рассмотренные модели имеют свои преимущества и недостатки, связанные, с одной стороны, с возможностью учета факторов, влияющих на процесс деформирования льда, с другой - с простотой их применения.

Итак, мы можем сделать следующие выводы.

В большинстве случаев авторы не уделяют должного внимания подбору конститутивных моделей, подходящих для описания пластичных свойств льда. Когда они используются в качестве моделей разрушения, обоснование их выбора играет важную роль, поскольку предельная прочность льда также зависит от большого количество факторов. В рассмот-ренных работах различных авторов не учитываются такие особенности льда, как зависимость его свойств от температуры, скорости деформаций, наличия стесненных условий и структуры.

Такие модели пластичности, как модели Друкера-Прагера и Джонсона-Кука не могут в полной мере учесть анизотропные свойства льда, поскольку соответствующие критерии текучести не имеют зависимостей прочностных характеристик от направления нагрузки. В связи с этим рекомендуется применять данные модели только при описании поведения условно изотропного (зернистого) льда. При моделировании столбчатого льда, обладающего явно выраженной анизотропией свойств, применение моделей Друкера-Прагера и Джонсона-Кука нецелесообразно.

Модель Хилла и ортотропная модель пластичности имеют возможность учитывать анизотропные свойства материалов, что является значимым преимуществом при моделировании льда столбчатой структуры. Используемые в обеих моделях квадратичные критерии текучести настраиваются через коэффициенты, легко определяемые по результатам экспериментальных данных. Однако само выполнение данных экспериментов в случае такого материала, как лёд, является нетривиальной задачей, требующей учёта ранее описанных факторов.

Явным преимуществом модели Друкера-Прагера является зависимость критерия текучести от среднего (гидростатического) напряжения. Этот факт дает возможность учитывать сложное напряженно-деформированное состояние ледяных образований, формирующееся в связи со стесненными условиями на контакте взаимодействия с сооружением. Кроме того, нами предложен алгоритм подбора параметров модели, основанный на предопределенных значениях прочности льда.

В связи с сильной зависимостью прочностных характеристик льда от его структуры рекомендуется разделять тело моделируемого ледяного образования на условно изотропный и ортотропный лёд. При этом модели пластичности, имеющие различный предел текучести в ортогональных направлениях (таких как ортотропная модель и модель Хилла), рекомендуется 
применять только для моделирования ортотропного льда, например столбчатой структуры. Это связано с отсутствием зависимости данных критериев от среднего (гидростатического) напряжения, а также с одинаковым пределом текучести на растяжение и сжатие.

Поскольку процесс взаимодействия льда с сооружениями сопровождается разрушением ледяных образований, то дальнейшим направлением исследований является анализ конститутивных моделей разрушения и развития повреждений и оценка их применимости в численном моделировании ледовых воздействий.

Вклад авторов в статью: А.Т. Беккер - постановка цели и задач исследования, описание предложенного алгоритма для определения параметров модели Друкера-Прагера, формирование выводов; O.А. Макаров - анализ конститутивных моделей программного комплекса SIMULIA Abaqus; Д.3. Гоголадзе - анализ конститутивных моделей программного комплекса ANSYS.

\section{СПИСОК ЛИТЕРАТУРЫ}

1. Ли Лян. Ледовая нагрузка на гидротехнические сооружения с наклонной гранью: дис. ... канд. техн. наук. СПб., 2014. 136 с.

2. Политько В.А. Воздействие ледовых полей на одно- и многоопорные гидротехнические сооружения с вертикальной опорной частью: дис. ... канд. тех. наук. М., 2018. 142 с.

3. ANSYS Documentation. Mechanical APDL. Material reference. Chapter 4: Nonlinear material properties. ANSYS, Inc, 2020.

4. Gürtner A., Bjerkås M., Forsberg J., Hilding D. Numerical modelling of a full scale ice event. Proceedings of $20^{\text {th }}$ IAHR Intern. Symposium on Ice. Lahti, Finland, June 14-17, 2010. Local Organising Committee of the $20^{\text {th }}$ IAHR International Symposium on Ice, 2010. URL: https://www.researchgate.net/publication/282330043_Numerical_modelling_of_a_full_scale_ice_even $\mathrm{t}-27.04 .2020$.

5. Gürtner A., Bjerkås M., Kühnlein W., Jochmann P., Konuk I. Numerical Simulation of Ice Action to a Lighthouse. Proceedings of the ASME $28^{\text {th }}$ Intern. Conference on Ocean, Offshore and Arctic Engineering. Honolulu, Hawaii, May 31-June 5, 2009. ASME, 2009. URL: https://www.researchgate.net/publication/268295189_Numerical_Simulation_of_Ice_Action_to_a_Lig hthouse - 27.04.2020.

6. Hilding D., Forsberg J., Gürtner A. Simulation of ice action loads on offshore structures. Proceedings of 8th European LS-DYNA Users Conference. Strasbourg, France, May, 2011. DYNAmore GmbH, 2011. URL: https://www.dynalook.com/conferences/8th-european-ls-dyna-conference/session-17/Session17_Paper5.-pdf - 27.04.2020.

7. Hilding D., Forsberg J., Gürtner A. Simulation of Loads from Drifting Ice Sheets on Offshore Structures. Proceedings of $12^{\text {th }}$ International LS-DYNA Users Conference. Detroit, USA. Livermore Software Technology Corporation, 2012.

8. Hill R. The Mathematical Theory of Plasticity. Oxford Univ. Press, 1983, 355 p.

9. Johnson G.R., Cook W.H. Fracture Characteristics of Three Metals Subjected to Various Strains, Strain rates, Temperatures and Pressures. Engineering Fracture Mechanics. 1985;21(1):31-48.

10. Nisja H.A. Numerical Modelling of Brittle Failure in Ice Structures: master's thesis. Norwegian Univ. of Science and Technology, 2014, $124 \mathrm{p}$.

11. Sand Bjørnar. Nonlinear finite element simulations of ice forces on offshore structures: doctoral thesis. Luleå Univ. of Technology, 2008, $264 \mathrm{p}$.

12. Schulson E.M., Duval P. Creep and fracture of ice. Cambridge Univ. Press, 2009, 417 p.

13. Sodhi D.S., Haehnel R.B. Crushing Ice Forces on Structures. J. of Cold Regions Engineering. 2003;17(4):153-170.

14. Sultabayev A.E. Development Concept for the Northern Caspian Sea: master's thesis. Univ. of Stavanger, Faculty of Science and Technology, 2015, $147 \mathrm{p}$.

15. The Abaqus documentation. Abaqus. Materials. Inelastic Mechanical Properties. Other plasticity models. Extended Drucker-Prager models. Dassault Systèmes Simulia Corp., 2019. 
FEFU: SCHOOL of ENGINEERING BULLETIN. 2020. N 2/43

Hydraulic Engineering www.dvfu.ru/en/vestnikis

DOI: http://www.dx.doi.org/10.24866/2227-6858/2020-2-15

Makarov O., Bekker A., Gogoladze D.

OLEG MAKAROV, MS student, Junior Researcher, International Research and Education Center R\&D Center Arctic, ORCID: https://orcid.org/0000-0003-3319-4481, e-mail: makarov.oa@dvfu.ru

ALEXANDER BEKKER, Doctor of Engineering Sciences, Professor, Scientific Adviser, School of Engineering, ResercherID: I-6494-2017, ScopusID: 35610298200, ORCID: http://orcid.org/0000-0002-2899-7995, e-mail: bekker.at@dvfu.ru DENIS GOGOLADZE, MS student, Junior Researcher, International Research and Education Center R\&D Center Arctic, ORCID: https://orcid.org/0000-0001-5497-807X, e-mail: gogoladze.dzu@dvfu.ru School of Engineering Far Eastern Federal University Vladivostok, Russia

\title{
Analysis of constitutive plasticity models in relation to numerical modelling of ice impacts
}

\begin{abstract}
In recent years, a large number of numerical experiments have been performed to estimate the magnitude of the impact of ice on offshore structures, with a variety of used material models. However, there are relatively few correct substantiations for using such models. In this regard, the question of reliability of the behavior of the modeled ice undergoing significant deformations remains open. Although in many software systems it is possible to integrate side user-defined models designed to describe the behavior of materials under load, the use of this tool requires the user to apply additional knowledge and skills. In this regard, this paper analyzes the constitutive models of plasticity of the material available in the ANSYS and SIMULIA Abaqus software packages which properly consider various factors that have a significant effect on the strength characteristics of ice. For these models, the analysis of parameters affecting the criterion of the onset of plastic flow is carried out. Conclusions are made on the possibility and advisability of using the considered models for various conditions. A review of numerical studies of the processes of interaction in the icestructure system performed by various authors, regarding the applied constitutive models, is carried out.
\end{abstract}

Keywords: constitutive models, ice impacts, ice plasticity, strain rate, review.

\section{REFERENCES}

1.Li Liang. Ice load on sloping hydraulic structures: dis. ... Candidate of Technical Sciences. St. Petersburg, 2014. $136 \mathrm{p}$.

2.Politko V.A. The impact of ice fields on vertical hydraulic structures with single and multiple supports: dis. ... Candidate of Technical Sciences. Moscow, 2018. 142 p.

3.ANSYS Documentation. Mechanical APDL. Material reference. Chapter 4: Nonlinear material properties. ANSYS, Inc, 2020.

4.Gürtner A., Bjerkås M., Forsberg J., Hilding D. Numerical modelling of a full scale ice event. Proceedings of 20th IAHR Intern. Symposium on Ice. Lahti, Finland, June 14-17, 2010. Local Organising Committee of the 20th IAHR International Symposium on Ice, 2010. URL: 
https://www.researchgate.net/publication/282330043_Numerical_modelling_of_a_full_scale_ice_event _ 27.04.2020.

5.Gürtner A., Bjerkås M., Kühnlein W., Jochmann P., Konuk I. Numerical Simulation of Ice Action to a Lighthouse. Proceedings of the ASME 28th Intern. Conference on Ocean, Offshore and Arctic Engineering. Honolulu, Hawaii, May 31-June 5, 2009. ASME, 2009. URL: https://www.researchgate.net/publication/268295189_Numerical_Simulation_of_Ice_Action_to_a_Lighthou se -27.04 .2020 .

6. Hilding D., Forsberg J., Gürtner A. Simulation of ice action loads on offshore structures. Proceedings of 8th European LS-DYNA Users Conference. Strasbourg, France, May, 2011. DYNAmore GmbH, 2011. URL: https://www.dynalook.com/conferences/8th-european-ls-dyna-conference/session-17/Session17-_Paper5.pdf-27.04.2020.

7.Hilding D., Forsberg J., Gürtner A. Simulation of Loads from Drifting Ice Sheets on Offshore Structures. Proceedings of 12th International LS-DYNA Users Conference. Detroit, USA. Livermore Software Technology Corporation, 2012.

8.Hill R. The Mathematical Theory of Plasticity. Oxford Univ. Press, 1983, 355 p.

9.Johnson G.R., Cook W.H. Fracture Characteristics of Three Metals Subjected to Various Strains, Strain rates, Temperatures and Pressures. Engineering Fracture Mechanics. 1985;21(1):31-48.

10. Nisja H.A. Numerical Modelling of Brittle Failure in Ice Structures: master's thesis. Norwegian Univ. of Science and Technology, 2014, 124 p.

11. Sand Bjørnar. Nonlinear finite element simulations of ice forces on offshore structures: doctoral thesis. Luleå Univ. of Technology, 2008, 264 p.

12. Schulson E.M., Duval P. Creep and fracture of ice. Cambridge Univ. Press, 2009, 417 p.

13. Sodhi D.S., Haehnel R.B. Crushing Ice Forces on Structures. J. of Cold Regions Engineering. 2003;17(4):153-170.

14. Sultabayev A.E. Development Concept for the Northern Caspian Sea: master's thesis. Univ. of Stavanger, Faculty of Science and Technology, 2015, 147 p.

15. The Abaqus documentation. Abaqus. Materials. Inelastic Mechanical Properties. Other plastici-

ty models. Extended Drucker-Prager models. Dassault Systèmes Simulia Corp., 2019. 